A)

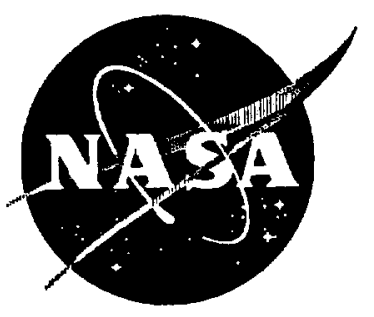

\title{
FUTURE HORIZONS IN X-RAY ASTRONOMY
}

Nicholas E. White
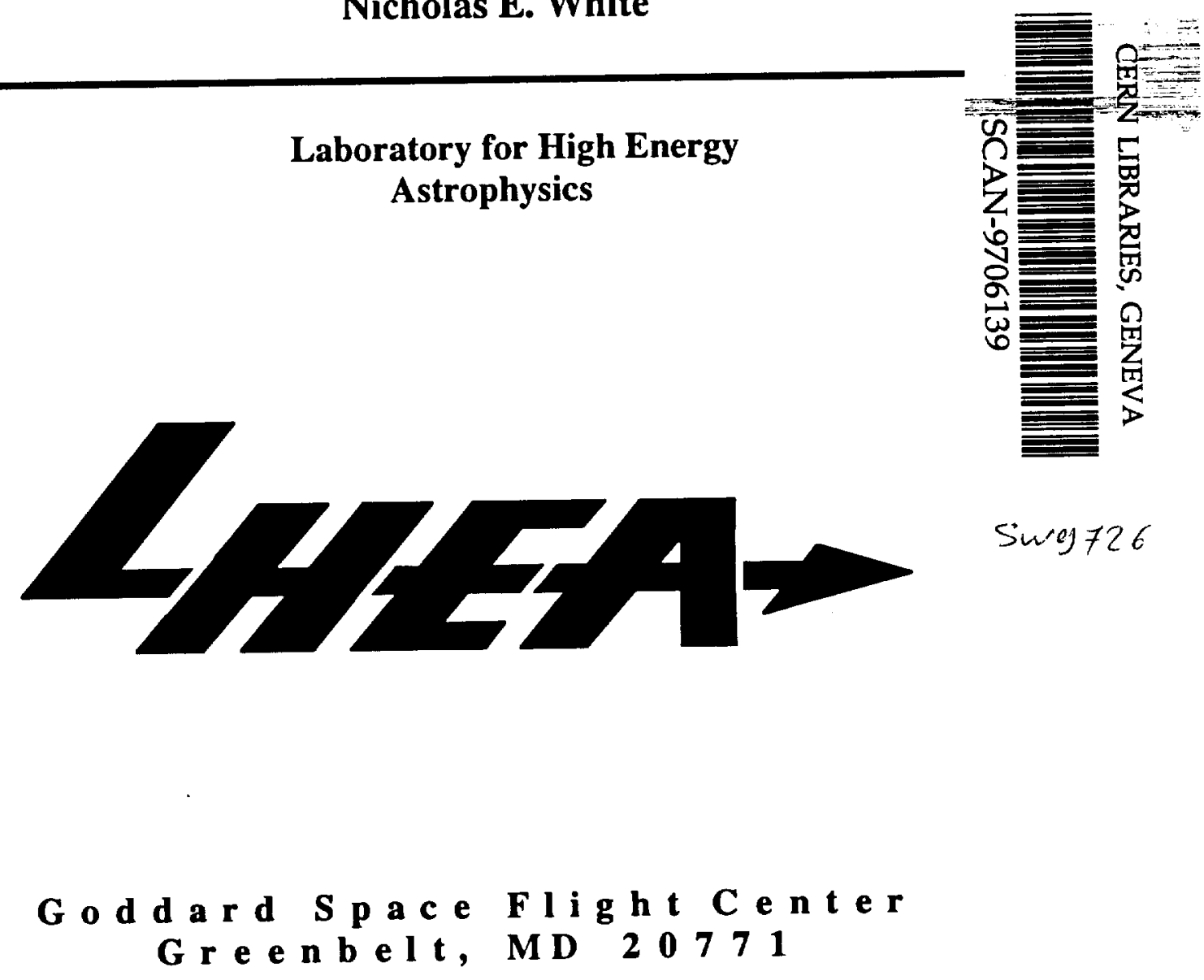

$\sin 9726$

Greetbelt, MD 20771 


\title{
FUTURE HORIZONS IN X-RAY ASTRONOMY
}

\author{
Nicholas E. White \\ Code 662, Laboratory for High Energy Astrophysics \\ NASA Goddard Space Flight Center \\ Greenbelt, MD 20771 \\ 301-286-8443
}

Accepted for publication in Proceedings Of The Space Technology \& Applications International Forum (Staif-97), October 1996. 


\title{
FUTURE HORIZONS IN X-RAY ASTRONOMY
}

\author{
Nicholas E. White \\ Code 662, Laboratory for High Energy Astrophysics \\ NASA Goddard Space Flight Center \\ Greenbelt, MD 20771 \\ 301-286-8443
}

\begin{abstract}
Some possible X-ray astronomy missions that may follow the currently approved missions during the first decade of the 21 st century are reviewed. These include a high throughput, broadband, and high resolution $X$-ray spectroscopy mission; a deep all-sky X-ray survey; a wide field of view X-ray telescope; a spectroscopic survey of the hot interstellar medium; and a next generation all-sky monitor.
\end{abstract}

\section{INTRODUCTION}

Over the relatively short 30-year history of X-ray astronomy, there has been a rapid evolution in instrumental capabilities. The first instruments flown on sounding rockets in the 1960 s and then subsequently on small satellites in the early to mid-1970s used simple mechanically collimated proportional counters and discovered several hundred cosmic sources, including the famous black hole candidate in our Galaxy, Cygnus X-1, and the first extragalactic sources. A major breakthrough in the field came with the launch by NASA of the HEAO-2 (Einstein) Observatory in 1978. This mission flew the first focusing X-ray telescope and the increased sensitivity showed the sky to be alive with $\mathrm{X}$-ray sources. This marked a watershed in the field when it became apparent that X-ray astronomy would have a major impact on all areas of astronomy. The recent German/US/UK ROSAT mission has made the first survey of the X-ray sky using focusing optics and has catalogued $\sim 100,000$ sources. The Japanese/US ASCA mission launched in 1993 carried the first CCD spectrometers and has begun the first extensive high quality spectroscopy of these sources. The NASA XTE mission launched at the end of 1995 is making the highest time resolution observations. The Italian-Dutch SAX mission launched in 1996 is providing the first sensitive X-ray broad band capability. At the turn of the century four major X-ray observatories will be launched. These are the US AXAF Observatory; the European XMM Observatory; from Russia (with extensive international collaboration), the Spectrum-X Gamma mission; and from Japan, the Astro-E mission. Collectively these missions will provide a wealth of detailed imaging and spectral information on a diverse array of cosmic sources. The currently planned observatories will only "scratch the surface" of the full exploitation of X-ray spectroscopy to address outstanding astrophysical questions. Substantial improvements will still be necessary in effective area, spectral resolution, and spectral bandpass to reach the fainter X-ray sources. In addition, we also expect significant X-ray astronomy missions within the current SMEX and MIDEX programs that will be focused on more specific science problems (e.g., all-sky surveys).

\section{CURRENT PLANS AND FUTURE NEEDS}

The workhorse instruments on the three major new X-ray observatories AXAF, XMM, and Astro-E that begin operation around the turn of the century are CCD cameras that provide a spectro-imaging capability with an energy resolution of $\sim 100 \mathrm{eV}$ over the $0.4-10 \mathrm{keV}$ band. These will produce tens of thousands of high quality CCD X-ray spectra from objects of all classes. All of these missions carry higher spectral resolution devices (gratings on XMM and AXAF and a micro-calorimeter on Astro-E) and these will gather detailed spectra of the brighter sources. The two sets of gratings on AXAF have $R \sim 500-1000$ from $0.1-2.0 \mathrm{keV}$, with an effective area ranging from 3 to 180 $\mathrm{cm}^{2}$. The peak effective area of the reflection gratings on $X M M$ is slightly higher, $-280 \mathrm{~cm}^{2}$ and covers the energy range 0.3 to $2.0 \mathrm{keV}$, with $\mathrm{R}=\mathrm{E} / \Delta \mathrm{E}=200-800$. The $\mathrm{X}$-ray Spectrometer, $\mathrm{XRS}$, originally to be flown on AXAF and now to be included as part of the Japanese Astro-E mission, utilizes an array of micro-calorimeters with an energy resolution of $10 \mathrm{eV}$ across the $0.3-8 \mathrm{keV}$ band, which includes the $\mathrm{K}$ lines of most of the important medium$\mathrm{Z}$ elements. The XRS has superior spectral resolution to the AXAF gratings above $3 \mathrm{keV}$ and, with a relatively 
constant effective area of $\sim 300 \mathrm{~cm}^{2}$, is comparable to that of the AXAF CCD detectors. While these new capabilities offer a major increase in performance, they have limitations that must be addressed by a next generation $\mathrm{X}$-ray observatory.

The AXAF and XMM gratings cannot be used to study extended sources because the spatial extent of the X-ray emission will hamper their sensitivity to line emission by creating confusion between spatial and spectral features. This is unfortunate because supernova remnants and clusters of galaxies are among the most line-rich X-ray sources. The collecting areas of the grating spectrometers are factors of 5-10 less than the CCD cameras on their respective missions and will require long exposures of $10^{5}-10^{6} \mathrm{~s}$ to obtain high quality spectra, even on the brightest point sources. On AXAF the gratings must share the observing time with the imaging experiments and the Astro-E XRS has a limited 2-year lifetime because of the need for cyrogenic cooling. The current generation of imaging $X$ ray telescopes has an upper energy threshold of $10 \mathrm{keV}$ above which the effective areas drop precipitously. Observations above $10 \mathrm{keV}$ are currently limited to using collimators or masks that have spatial resolutions ranging from arcminutes to degrees. The large size of the detectors means that these observations have been severely limited by background. The first results from ASCA are showing that sensitive observations of the $>10 \mathrm{keV}$ band are necessary to constrain the continuum spectra of many classes of X-ray source. This is crucial in highly absorbed sources and sources where the Compton reflection of X-rays from cold material is present. Extended energy coverage is also necessary to differentiate between thermal and non-thermal emission. Typical observations using these powerful new observatories will have sensitivities several orders of magnitude below that of the ROSAT allsky survey. This means that many observations will effectively be made shooting in the dark. In addition, many $\mathrm{X}$ ray sources are variable. A sensitive all-sky monitor is needed to study and characterize this activity. This monitor can alert the major space and ground based observatories to unusual events.

\section{THE HIGH THROUGHPUT X-RAY SPECTROSCOPY MISSION}

In September 1994, NASA issued NRA 94-OSS-15 to solicit proposals for concept studies of new astrophysics flight missions and selected a number of concepts for study, including "The Next Generation X-ray Observatory" (PI, Nicholas White of GSFC) and the "Large Area X-ray Spectroscopy Mission" (PI, Harvey Tananbaum of SAO). Given the similarities of the science objectives emphasizing high throughput, high resolution X-ray spectroscopy, the two teams agreed to pursue a single study of a common mission named the High Throughput $X$-ray Spectroscopy (HTXS) mission. Most recently, the PI (Paul Gorenstein of SAO) of a third selected mission concept joined the HTXS team merging those areas of his study most similar to HTXS in science objectives and technical approach. Following these steps, the expanded team became a widely-based Science Working Group (SWG) reporting to NASA Headquarters. With its increased capabilities, HTXS will address many fundamental astrophysics questions such as the origin and distribution of the elements from carbon to zinc, the formation and evolution of clusters of galaxies, the validity of general relativity in the strong gravity limit, the evolution of supermassive black holes in active galactic nuclei, the details of supernova explosions and their aftermath, and the mechanisms involved in the heating of stellar coronae and driving of stellar winds.

Figure 1 shows the effective area curves and resolution for the baseline mission. The HTXS science objectives require a factor of 20-100 increase in sensitivity across the $0.25-40 \mathrm{keV}$ band, which requires an effective area using a grazing incidence $X$-ray system of at least $15,000 \mathrm{~cm}^{2}$ at $1 \mathrm{keV}, 6,000 \mathrm{~cm}^{2}$ at $6.4 \mathrm{keV}$, and $1500 \mathrm{~cm}^{2}$ at 40 $\mathrm{keV}$. It is not practical to cover the entire $0.25-40 \mathrm{keV}$ band with a single telescope/instrument combination and the baseline design requires a matched set of high throughput focusing telescope systems to cover simultaneously the low and high energy bands. The low energy system is a spectroscopy X-ray telescope (SXT) that is optimized to maintain a spectral resolving power of at least 300 across the $0.25-12 \mathrm{keV}$ bandpass. The angular resolution of the SXT is $15^{\prime \prime}$ half power diameter HPD. A hard X-ray telescope (HXT) system provides the required effective area between 10 and $40 \mathrm{keV}$ (and possibly up to $100 \mathrm{keV}$ ) with a spatial resolution of 1' HPD. 


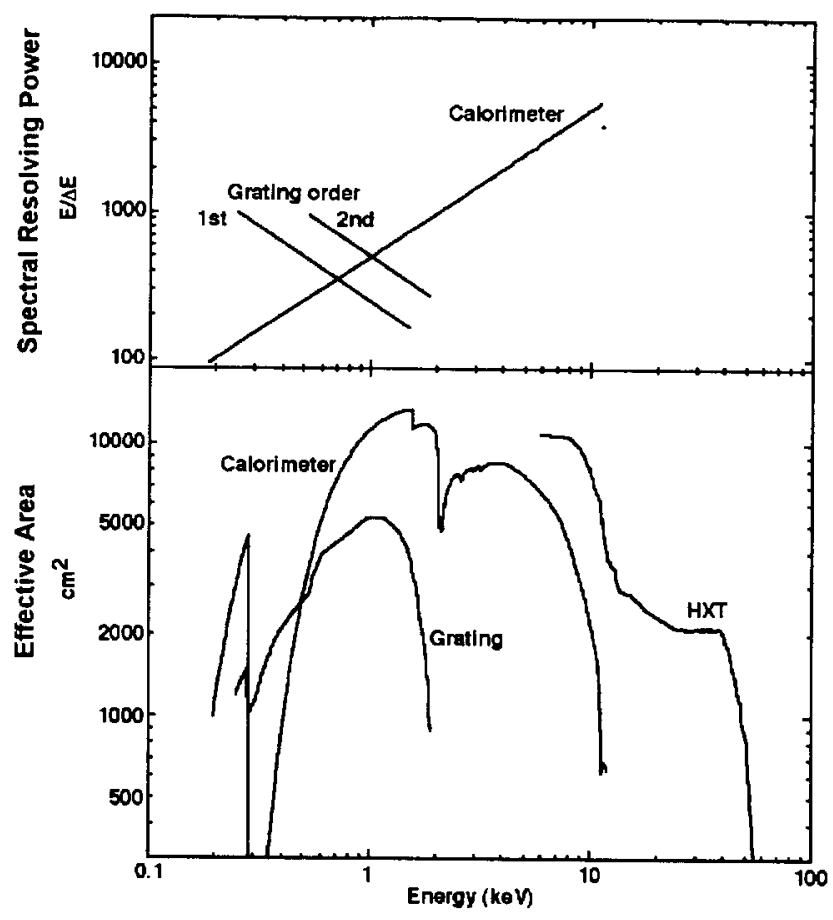

Figure 1. Effective Area Curves and Resolution for the HTXS Baseline Mission.

To increase the telescope collecting area requires either an X-ray telescope with very large aperture and very long focal length, or an approach utilizing several mirror modules each with its own spectrometer/detector system. The technical challenges and cost associated with a single big telescope appear prohibitive at this time and utilizing multiple telescope modules is more cost effective. The HTXS study team has taken this process one step further by recognizing that several small spacecraft and modest launch vehicles (e.g., the Delta II) each carrying one "science unit" can cost less than one very large spacecraft and launcher (e.g., Titan IV) carrying the entire HTXS mission. The baseline HTXS concept is to build six identical modest satellites in "assembly-line" style. This approach is very robust in that risks are distributed over several launches and several spacecraft with no single failure leading to loss of mission. The choice of a relatively distant libration point orbit allows simple operational scenarios with all satellites available to point at the selected target with high viewing efficiency (no trapped radiation belts or earth occultations). With such an orbit and a relatively benign environment, the spacecraft can be simpler, lighter, and less costly. The intent is that this mission would be fully capable about nine years after the launch of AXAF, around 2007. Assuming a launch interval of three months, this would require the first launch to take place in mid-2005.

Light-weight replicated mirrors capable of providing the required HTXS large collecting area and modest angular resolution (15" compared to AXAF's 0.5 ") are being developed. Replicated shells using silicon carbide, cyanate ester, and thin-walled rib-reinforced nickel carriers are in-process with the intent of producing individual assemblies having an X-ray collecting area of $\sim 5000 \mathrm{~cm}^{2}$ at a weight of order $250 \mathrm{~kg}$. Replicated foils already can provide comparable areas with a weight of $200 \mathrm{~kg}$ and modified manufacturing and assembly approaches are under evaluation for improving the angular resolution of these optics to the desired level. The SXT uses two complimentary spectrometer systems to achieve the desired energy resolution: 1) an array of high efficiency quantum micro-calorimeters with a resolution of $2 \mathrm{eV}$, and 2) a reflection grating with resolution of $\Delta \lambda$ of $0.05 \AA$ in first order and $0.025 \AA$ in second order along with a CCD readout. The gratings deflect part of the telescope beam away from the calorimeter array. The two spectrometers are complimentary with the grating optimal for high resolution spectroscopy at low energies and the calorimeter at high energies (Figure 1). The gratings also provide coverage in the $0.3-0.5 \mathrm{keV}(25-40 \AA)$ band, which is essential because the calorimeter light-blocking filters cause a loss of response around the carbon K-edge (Figure 1). This is particularly important for high redshift objects where line rich regions will be moved down into this energy band. To cover the bandpass up to $40 \mathrm{keV}$ with appropriate sensitivity requires focusing optics to place a large fraction of the flux collected by a large area telescope onto a 
relatively small spot in the focal plane. The reduction in particle and sky background in a focusing system represents a significant advance in sensitivity as well as a major reduction in the weight and cost of the instrumentation. The following three approaches are being considered: 1) Nested Wolter-I or conical mirror shells coated with graded multilayers to enhance the high-energy reflectivity; 2) Small-radius, tightly-nested Wolter-I or conical mirror shells which achieve high-energy reflectivity by utilizing very low graze-angles; and 3) Curved microchannel plate detectors that concentrate the beam using an array of capillaries. The advantage of the first approach is that it is likely to yield a telescope with response extending to significantly higher energies than the specified $40 \mathrm{keV}$; the advantage of the second is that it may potentially yield optics of lower total mass; and the advantage of the third is that it could yield high effective areas at low mass.

\section{SURVEY MISSIONS}

The ROSAT all-sky survey has provided the first detailed view of the sky in the $0.1-2 \mathrm{keV}$ band and this has resulted in the detection of about 40,000 sources. Some 80,000 sources were detected during the pointed phase of the mission when about $10 \%$ of the sky was covered with varying degrees of sensitivity. In 1999 the German ABRIXAS small satellite will be launched to perform a survey in the $0.5-10 \mathrm{keV}$ band. Previous surveys in this band have been limited to collimated experiments with fields of view of order 1 degree (e.g., HEAO-1) and have been confusion limited resulting in only $\sim 1000$ sources. The ABRIXAS mission will use focusing optics to obtain a more sensitive view in this energy band. The ABRIXAS optical system consists of seven identical Wolter-I telescopes of $1.6 \mathrm{~m}$ focal length, each with 27 nested. The seven focal planes share one $6 \mathrm{~cm} \mathrm{x} 6 \mathrm{~cm} \mathrm{XMM} \mathrm{pn-CCD}$ array. The optical axes diverge and the seven fields of view, each about 40 arcminutes in diameter, are approximately seven degrees apart from each other on the sky, which is scanned along great circles. One main goal of the project is to study the absorbed AGN population and its contribution to the X-ray background. It is expected to detect 10,000-20,000 new AGN. ABRIXAS will also obtain high-quality spectra of diffuse emission objects like clusters and supernova remnants.

The sources detected by ROSAT and expected to be seen by ABRIXAS represent the tip of an iceberg. If an X-ray survey can reach an order of magnitude deeper, to $\sim 10^{-14} \mathrm{erg} \mathrm{cm}^{-2} \mathrm{~s}^{-1}$, then the source density would be $\sim 100$ per square degree. Such a deep X-ray all-sky survey would produce of order 4 million sources and would be a very timely addition to the large mega-source surveys being conducted in the optical and IR (e.g., the Sloan Survey). A deep X-ray survey would be dominated by X-ray emitting AGN and would allow for the first time the use of X-ray sources to trace the large scale structure of the universe over a wide range of redshift. The PEP (present epoch plus) mission concept (PI: Keith Jahoda, GSFC) combines an X-ray imaging survey with a collimated proportional counter system. The sources detected in the imaging system are subtracted from the X-ray background emission detected in the collimated system. The residuals can then be used to trace the distribution of the fainter unresolved sources in the X-ray background. An order of magnitude scaling from ROSAT shows that for a survey to reach $\sim 10$ times deeper than ROSAT in a comparable survey time (six months) will require a combination of a larger field of view or higher collecting area. At the extreme this would require a field of view of $\sim 6$ degrees, or ten times higher collecting area. This would be within the capabilities of a NASA MIDEX mission (using lightweight X-ray optics). For example, taking 28 of the foil optic telescopes that were flown on ASCA and canting each telescope to provide a larger effective field of view would exceed this requirement and provide a powerful sky survey. The current angular resolution of the foil optic telescopes of 1 arcminute half power diameter is probably not sufficient for such a deep survey and at least a factor of 2 improvement would be required.

An X-ray wide field survey (an "X-ray Schmidt" Telescope) covering the band from 0.1 to $2 \mathrm{keV}$ with a large field of view and an angular resolution of about 2 arcseconds would provide a complimentary survey capability. Deep surveys could be carried out and the candidate optical counterparts to X-ray sources identified unambiguously. Such a mission could efficiently search for distant clusters of galaxies over several hundreds of square degrees using the extent of the X-ray emission to uniquely identify cluster sources. Both the deep and the cluster surveys would detect large numbers of AGN which could be used as a flux limited sample.

The immediate solar neighborhood (i.e., within $100 \mathrm{pc}$ ) is filled with a hot, tenuous gas which produces substantial thermal soft X-ray emission below $1 / 4 \mathrm{keV}$. This gas has a substantial thermal pressure and is a dominant factor in the energy budget of the local ISM. The soft X-ray band is the best waveband in which to observe this hot gas. 
Major questions remain to be answered about the hot ISM including the temperature distribution, emission measure, ionization distributions, and metallicity of the gas. X-ray spectroscopic observations with resolution sufficient to measure line strengths, ionization states, and atomic abundance ratios are necessary to answer these questions. Such observations are necessary to discern the history of the solar neighborhood. The Hot Interstellar Medium Spectrometer (HIMS) mission (PI: Wilt Sanders, University of Wisconsin) is a SMEX or MIDEX class mission designed to answer the above questions. HIMS will make a high resolution spectroscopic all-sky survey of diffuse emission in the 50-1000 eV band using an X-ray micro-calorimeter with 2-5 eV resolution. In the SMEX version, this provides a complete sky survey with a field of view of a few degrees dictated by a simple collimator. In the MIDEX version, a telescope is added to provide larger collecting area and the ability to resolve features on a 4 arcminute scale.

\section{NEXT GENERATION ALL-SKY MONITORS}

There has been considerable success in monitoring the brightest $100 \mathrm{X}$-ray sources in the sky using all-sky monitors. The first of these were a pin hole camera flown on Ariel $V$ and large field of view collimated detectors on the Vela 5B satellite. These detectors operated during the late 1960s and 1970s. They demonstrated that such monitors could provide both alerts to transient events and a record for investigations of the long term variability of X-ray sources. The XTE ASM, CGRO BATSE, the wide field camera on SAX, and MOXE on the upcoming Spectrum-X Gamma provide the current capability. The current generation of ASM utilize either masks or wide field of view slats and are confusion limited. They can reach only the bright X-ray binary sources and a few of the brightest AGN. A next generation ASM must be able to reach at least one order of magnitude deeper to reach several thousand AGN and study their variability on timescales of hours to months. Another limitation of the current ASMs is that they only operate in the $>2 \mathrm{keV}$ band. The $0.1-2 \mathrm{keV}$ band has never been continuously monitored and yet there are expected to be many interesting variable $\mathrm{X}$-ray sources in this band, including coronal activity from nearby stars and accreting white dwarf systems.

Angel (1979) proposed that by using curved square channel arrays, it would be possible to mimic the visual system of a macruran crustacean (lobsters, shrimps, and crayfish) and provide a wide field of view optic. Recent advances in the development of micro-channel plate (MCP) devices by curving them into a spherical geometry and using square profile channels may realize the Angel Lobster-eye telescope for X-ray astronomy. Recent work at the University of Leicester by George Fraser and at the University of Melbourne by Keith Nugent and Andrew Peele has shown that the MCP array is rapidly evolving to becoming a useful and practical X-ray optic. X-rays are brought to a focus with an angular resolution comparable to the angle that an individual channel subtends at the detector. As a true focusing device, the Lobster-eye optic can image objects effectively against the diffuse sky background and so allows the observation of faint objects. The optic consists of an array of square channels. Those $\mathrm{X}$-rays that reflect off two orthogonal walls are sent to a common focal spot. Those that only hit one wall end up on a line and the remainder pass straight through. About $25 \%$ of the $\mathrm{X}$-rays end up in each component. The image of a point source takes the form of a distinctive cruciform shape which was first demonstrated by Fraser and more recently by Peele and Nugent. A Lobster-eye optic is capable of a field of view covering the entire sky, but this is impractical as it would require a spherical lens and detector. As discussed by Priedhorsky, Peele, and Nugent (1996) it is better to build the instrument out of distinct modules each with a field of view of 1/4 sr (Figure 2). The entire coverage can be obtained by packing eight telescopes into a cylindrical bay on a SMEX (Figure 2). By spinning the satellite, the entire sky coverage can be obtained. The total effective collecting area is only about $1 \mathrm{~cm}^{2}$ (comparable to MOXE); but the focusing capability provides a much more sensitive instrument capable of monitoring about a thousand AGN, a thousand stars, a few hundred cataclysmic variables, and reaching the ROSAT survey limit within one week.

Another approach being pursued by Gorenstein et al. (1996) is a telescope composed of two similar orthogonal stacks, each made from equally spaced identical flats that reflect on both faces. Its advantages are compatibility with large aperture and large field of view. They are attempting to improve the flatness and reflectivity of an inexpensive, thin, commercial glass by replicating a smoother, flatter, gold coated surface on both faces. A small 1D mirror assembly covering a five-degree field of view was constructed from inexpensive, thin commercial glass that was coated on two sides with nickel. X-ray testing indicated that its resolution is consistent with theory and the approximately two arcminute deviation from flatness of the glass. At GSFC a similar approach is being investigated 
by W. Zhang and R. Petre using Mylar foils stretched and held in a fixture. The GSFC team is also investigating the relative merits of $1 \mathrm{~d}$ vs. $2 \mathrm{~d}$ focusing optics for ASM applications.

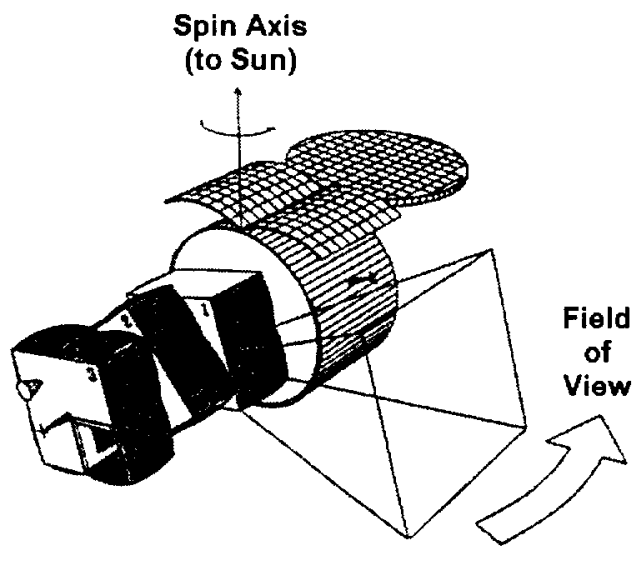

\section{Telescope Layout (bay 1)}

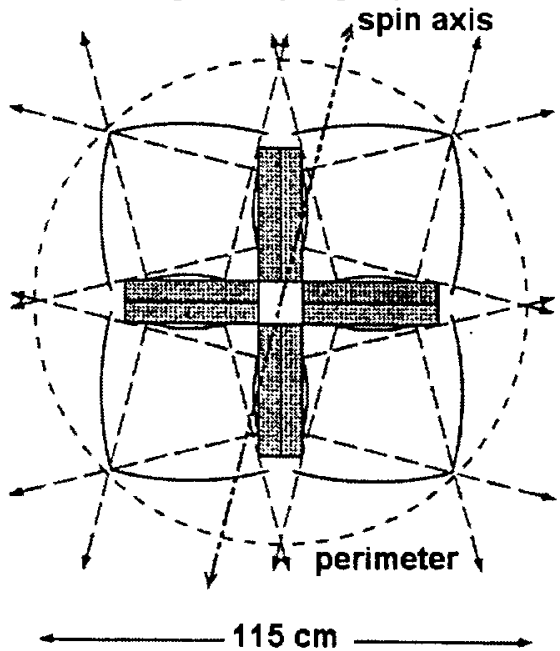

Figure 2. Possible Configuration for Lobster-eye ASM Packaged into NASA SMEX.

\section{CONCLUSIONS}

The HTXS mission has already emerged as the likely successor to AXAF in the NASA long-term program. Deeper all-sky surveys would be a natural precursor to such a mission. A Lobster ASM will provide a new view on the time variability of many classes of objects as well as a new sensitive alarm to transient events in the soft X-ray band. A spectroscopic survey of the hot interstellar medium is necessary to resolve its origin. The detailed character of these mission concepts may evolve over the coming years, but their underlying science focus and capabilities will remain the same. While none of the mission concepts discussed have yet been approved, it seems likely that some or all will be developed at some point over the coming years.

\section{Acknowledgments}

I thank Harvey Tananbaum, Neil Gehrels, Fiona Harrison, Keith Jahoda, Steve Kahn, Richard Kelley, Frank Marshall, Andrew Peele, Rob Petre, William Priedhorsky, Caroline Stahle, and W. Zhang for discussions.

\section{References}

Angel, J.R.P. (1979) "Lobster Eyes as X-ray Telescopes," ApJ., 233:364-373.

Gorenstein, P., et al. (1996) "Lobster-eye x-ray telescope prototype," Proc. SPIE 2805:74-80.

Holt, S.S. and W.C. Priedhorsky (1987) “All-sky monitors for X-ray astronomy," Sp. Sci. Rev. 45:269-289.

Priedhorsky, W.C., A. Peele, and K. Nugent (1996) "An X-ray all-sky monitor with extraordinary sensitivity," MNRAS 279:733-750. 